\title{
Metodología de análisis por medio de simulación de la modulación M-QAM sobre un canal afectado por desvanecimiento y efecto Doppler
}

\author{
Camilo Torres Zambrano \\ M.Sc. en Ingeniería Electrónica, \\ Pontificia Universidad Javeriana \\ Ingeniero de Tiempo Completo, Itansuka \\ Bogotá, Colombia \\ camilo.torresza@ecopetrol.com.co
}

\author{
Carlos Iván Páez Rueda \\ M.Sc. en Ingeniería Eléctrica, Universidad de los Andes \\ Docente e Investigador, Pontificia Universidad Javeriana, \\ Director del grupo de investigación SISCOM \\ Bogotá, Colombia \\ paez.carlos@javeriana.edu.co
}

\begin{abstract}
Resumen- En el artículo se presenta la metodología para determinar el desempeño de un sistema de comunicaciones digitales conformado por una modulación M-QAM (M-ary Quadrature Amplitude Modulation) afectado por un canal con desvanecimiento multi-trayectoria plano y lento tipo Rayleigh y un ruido Gassiano limitado en banda, por medio de la técnica de simulación por la envolvente compleja. Los resultados obtenidos son validados con algunas cotas de las probabilidades de error teóricas simplificadas para este tipo de modulaciones y canales. La metodología establece un procedimiento sistemático para encontrar las curvas de desempeño que caracterizan al sistema de comunicaciones bajo las condiciones no idealizadas del canal, las cuales permiten calcular los radio enlaces de los sistemas modernos como LTE (Long Term Evolution) y la televisión digital DVB-T.
\end{abstract}

Palabras clave—Canal Rayleigh, Envolvente Compleja, Desvanecimiento Multitrayectoria, Modelo Espectral de Jakes.

Abstract- In this paper the methodology for determining the performance of a digital communications system consisting of M-QAM modulation (M-ary Quadrature Amplitude Modulation) affected by a fading channel with multi-path Rayleigh slow, flat and a Gaussian noise band limited is presented by means of complex enveloped simulation technique. The results are validated with some simplified theoretical probability of error for this kind of modulations and channels. The methodology establishes a systematic procedure for finding the performance curves that characterize the communication system under non idealized conditions of channel, which allow the calculation of radio links to modern systems such as LTE (Long Term Evolution) and digital television DVB-T.

Keywords- M-QAM, Rayleigh Channel, Complex Envelop, fading, Jakes's Model.

\section{INTRODUCCIÓN}

La tecnología de las comunicaciones inalámbricas tiene un papel preponderante en la sociedad actual ya que facilita la comunicación en cualquier momento y cualquier lugar. Al utilizar el canal de radio frecuencia como medio de transmisión no guiado y no controlado en un sistema de comunicaciones, los esquemas de modulación deben combatir todos los fenómenos que en él se presentan, en particular, el desvanecimiento multi-trayectoria. Este fenómeno, cuya característica usualmente es variante en tiempo, es producido por la velocidad de movimiento del transmisor o del receptor o por la disposición variante de los objetos presentes en el canal, causantes de una reflexión, refracción, dispersión y retardo de la onda transmitida. La comunidad científica en [1][6], ha cuantificado el fenómeno multi-trayectoría en un sistema de comunicaciones y generado cotas de desempeño, cuya evaluación permite caracterizar un sistema de comunicaciones ante ese fenómeno con condiciones idealizadas.

Las diversas particularidades de cada sistema requieren eventualmente de la evaluación del desempeño ante estas condiciones variantes por medio de la simulación, ya que el modelo teórico usualmente es inmanejable por su complejidad. En este caso, la simulación por envolvente compleja es la técnica más utilizada ya que permite simular el sistema de comunicaciones con una frecuencia de muestreo sustancialmente inferior a cambio de una representación de funciones en el plano complejo [7]-[9].

Aquí se presenta la metodología formal de simulación por la envolvente compleja para el caso particular de la modulación M-QAM, el cual incluye el modelo de la señal modulada, el modelo del canal con ruido Gaussiano y el modelo de un canal variante. El aporte fundamental es precisar la metodología de simulación y análisis que incluyen el modelo variante en tiempo de tipo Rayleigh con 
un efecto Doppler moderado [2],[10], modelado a través del espectro Jackes. Los resultados de la evaluación de cada simulación son exhaustivamente analizados desde el punto de vista probabilístico y son comparados con algunas cotas encontradas en la literatura. Finalmente, con las curvas encontradas es posible evaluar el sistema en un enlace de comunicaciones moderno y buscar estrategias para mejorar su desempeño.

\section{METODOLOGÍA DE SIMULACIÓN}

\section{A. Envolvente compleja}

A una señal real de banda angosta $x(t)$ con componentes en frecuencia $f \in\left[f_{1}, f_{2}\right]$ se le puede asociar una señal analítica (1) denominada la preenvolvente compleja y denotada por $x_{a}(t)$, donde $x_{H}(t)$ es la transformada de Hilbert de $x(t)$. A la señal analítica $x_{a}(t)$ se le puede asociar otra señal denotada por $\tilde{x}(t)$ calculada por (2) y la cual recibe el nombre de la envolvente compleja.

$$
\begin{gathered}
x_{a}(t)=x(t)+j x_{H}(t) \\
\tilde{x}(t)=x_{a}(t) e^{-2 \pi \mathrm{f}_{0} t j}
\end{gathered}
$$

La envolvente compleja es una representación banda base de la señal original como se esquematiza en su transformada de Fourier en la Fig. 1 y, por ende, para su caracterización discreta requerirá de una cantidad mucho menor de muestras con respecto a la señal original. La expresión $e^{-2 \pi \pi_{0} t j}$ en la envolvente compleja representa la frecuencia promedio de la portadora.

\section{B. Transmisión Digital M-QAM}

En la Fig. 2, se detalla el diagrama en bloque conceptual que representa una transmisión digital que utiliza una modulación M-ary QAM, la cual es el objeto de análisis del presente trabajo.

El modulador digital M-QAM se encarga de transformar el bloque k-ésimo de bits de tamaño $L=\log _{2}(M)$, denotado por $\left\{x_{L k+1}, \ldots, x_{K L+L}\right\}$ en una señal análoga $S_{k}(t)$ denotada por (3), válida en el intervalo de tiempo $\left[k T_{s},(K+1) T_{s}\right]$, donde las amplitudes $d_{k}$ y $q_{k}$ dependen de la energía transmitida por el bloque k-ésimo y $f_{0}$ es la frecuencia central de operación.

$$
S_{k}(t)=A_{c} d_{k} \cos \left(2 \pi f_{0} t\right)-A_{c} q_{k} \sin \left(2 \pi f_{0} t\right)
$$

El diagrama en bloque denominado receptor, cuya función es extraer el vector que contiene la información necesaria para estimar cuál fue el símbolo enviado a partir de la señal recibida del canal $r_{k}(t)$ en el intervalo de tiempo $\left[k T_{S^{\prime}}(k+1) T_{s}\right]$.

FIG. 1. EQUIVALENTE PASABAJOS COMPLEJO DE UNA SEÑAL BANDA ANGOSTA
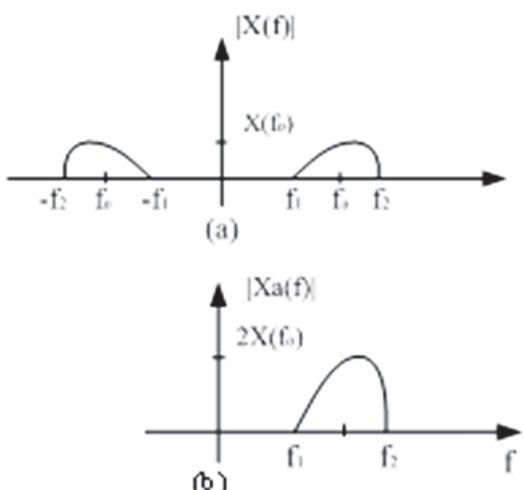

(b)

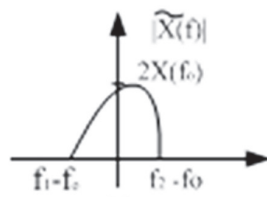

(c)

Fuente: los autores

FIG. 2. DIAGRAMA EN BLOQUES DEL MODULADOR, CANAL Y RECEPTOR M-QAM

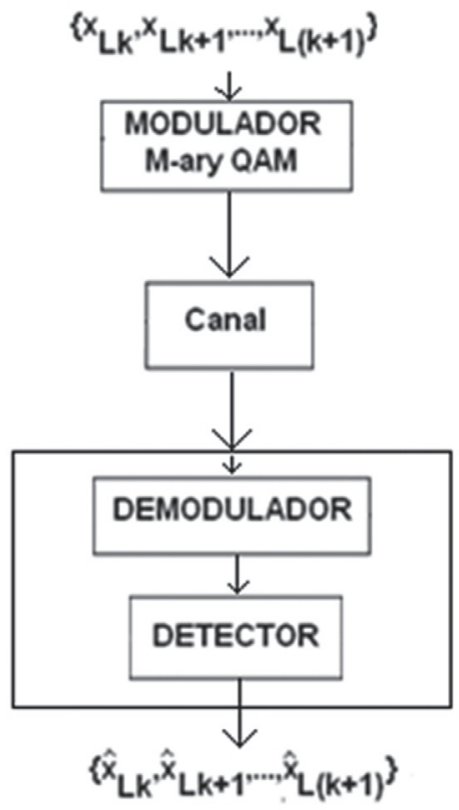

Fuente: los autores 
El objetivo del subsistema Demodulador es proyectar la señal recibida $r_{k}(t)$ en el sub-espacio de la modulación. Su resultado es un vector denotado por $r_{k}=\left[r_{k l}, \ldots r_{k L}\right]$, el cual representa la señal k-ésima enviada, que incluye los fenómenos introducidos por el canal. El objetivo del subsistema detector, es encontrar la secuencia de bits original asociado al k-ésimo bloque, minimizando la probabilidad de una incorrecta decisión. Diversos Criterios y algoritmos para implementar los subsistemas de Demodulador y Detección se pueden encontrar en [1],[4]-[5],[11].

\section{Canal AWGN}

El canal AWGN es un canal aleatorio Gaussiano, en el cual se genera una salida ideal modelada por medio de (4) en un intervalo de tiempo $\left[k T_{s}(k+1) T_{s}\right]$.

$$
r_{k}(t)=s_{k}(t)+n(t)
$$

Donde $n(t)$ es un proceso aleatorio que representa un ruido ideal denominado Blanco Gaussiano, conformado por una densidad espectral de potencia constante en todas las frecuencias de valor $N_{0} / 2$, con media cero e independiente de la señal de entrada. La señal $s_{k}(t)$ se asume que está limitada en frecuencia, con ancho de banda $W$ y con potencia finita. Aún lo anterior, el modelo de un canal AWGN no es conveniente para analizar sistemas desde el punto de vista de la causalidad y de potencia, ya que éste no es físicamente realizable. Por tal razón, se utilizará el ruido limitado en banda derivado del ruido blanco Gaussiano para el análisis del sistema. El canal Gaussiano con ruido limitado en banda es caracterizado por (5) en un intervalo de tiempo $\left[K T_{s},(k+1) T_{s}\right]$, donde las funciones $n_{d}(t)$ y $n_{q}(t)$ son procesos estocásticos banda base con ancho de banda $\mathrm{W}$, mutuamente independientes, con media cero y varianza $N_{0} W / 2$.

$r_{k}(t)=s_{k}(t)+n_{d}(t) \cos \left(2 \pi f_{0} t\right)-n_{q}(t) \sin \left(2 \pi f_{0} t\right)$

\section{Canal con Desvanecimiento Plano y Lento}

En el modelo de un canal de comunicaciones mediante el ruido Gaussiano por medio de (4)-(5), la señal de entrada solamente está afectada por una atenuación y retardo constante. Los canales afectados por desvanecimiento multitrayectoría generan atenuaciones y retardos variantes con el tiempo, efectos que pueden degradar significativamente el desempeño del sistema de comunicación y, por tanto, requiere de un análisis detallado [12]. Si un canal varía lentamente, esto es, se tiene una velocidad lenta de movimiento entre el transmisor y el receptor con respecto a la duración del símbolo, entonces éste puede ser modelado por medio de (6), donde $R$ y $\varphi$ son variables aleatorias distribuidas con parámetros estadísticos inalterados en el intervalo $\left[k T_{s},(k+1) T_{s}\right]$.

$$
r_{k}(t)=R \cdot s_{k}(t+\varphi)+n(t)
$$

En el caso particular del modelo con desvanecimiento plano y lento, el ruido Gaussiano es limitado en banda, la variable aleatoria $\varphi$ se atribuye como una variable uniformemente distribuida en el intervalo $[-\pi, \pi]$ y $R$ se distribuye como una variable aleatoria tipo Rice o Rayleigh. En caso de ser Rice, significa que el canal modela el fenómeno de múltiples trayectorias donde una tiene un componente de potencia dominante. En caso de utilizarse una variable aleatoria Rayleigh, el canal modela el fenómeno de múltiples vías donde no existe un componente dominante [13]-[14]. Si se asume, que el desvanecimiento es lo suficientemente lento con respecto al tiempo de convergencia del sistema de sincronismo del receptor, entonces la recepción coherente permitirá simplificar (6) a (7). En la ecuación (7), se evidencia que el modelo de un canal con desvanecimiento plano y lento, considera una atenuación aleatoria igualmente distribuida en un periodo de un símbolo.

$$
r_{k}(t)=R \cdot s_{k}(t)+n(t)
$$

Para analizar el escenario con movimiento lento desde el dominio de la frecuencia, es necesario considerar el efecto Doppler en la simulación de un canal variante con el tiempo. En [15] se describe analíticamente el modelo de Jakes, frecuentemente usado para describir las variaciones de un canal de radio enlace con las características planas y lentas. En este trabajo se asocia la variable $R$ a una señal banda base compleja, la cual es representada por un proceso aleatorio con media cero denotado por $\mu(t)=\mu_{1}(t)+j \cdot \mu_{2}(t)$. Donde, $\mu_{1}(t)$ y $\mu_{2}(t)$ son procesos banda angosta también banda base, con media cero y varianza $\operatorname{var}\left(\mu_{i}(t)=\sigma_{o}^{2}\right.$ para $i=1,2$. Estos procesos aleatorios frecuentemente resultan ser estadísticamente independientes, lo cual implica que la función de correlación cruzada es cero. En térmi- 
nos generales, un proceso aleatorio se describe completamente por su media y su función de autocorrelación (ACF). Para una antena receptora omnidireccional en un ambiente de dispersión isotrópica bidimensional, en [16] se demostró que la ACF de $\mu_{i}(t)$ se puede representar por medio de (8).

$$
A C F_{\mu 1, \mu 1}(\tau)=\sigma_{0} J_{0}\left(2 \pi f_{\max } \tau\right)
$$

Donde $f_{\max }$ es la máxima frecuencia Doppler y $J_{0}(\cdot)$ es la función de Bessel de primera clase de orden cero. Por lo tanto, la transformada de Fourier de (8) genera el espectro de potencia Doppler (9), la cual es conocida como espectro Jakes. La frecuencia máxima Doppler $\left(f_{\max }\right)$ depende de los escenarios que va a modelar. En la literatura se documenta dicho valor entre $1 \mathrm{~Hz}$ y $100 \mathrm{~Hz}$. Para valores mayores a $100 \mathrm{~Hz}$ se considera que el sistema de comunicaciones comienza a estar afectado por un desvanecimiento rápido [14].

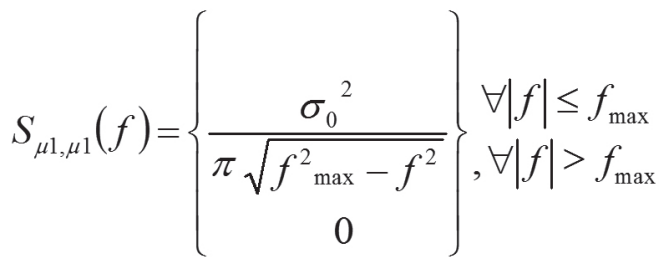

\section{E. Modelo por Envolvente Compleja de M-QAM}

Para M-QAM, la señal análoga se puede describir en un intervalo de un símbolo por medio de (10) y, por ende, su envolvente compleja tiene una representación sencilla dada por medio de (11).

$$
\begin{gathered}
s_{k}(t)=A_{c} \operatorname{Re}\left\{\left(d_{k}+j q_{k}\right) e^{-2 \pi f_{o} t j}\right\} \\
\widetilde{s}_{k}(t)=A_{c}\left(d_{k}+j q_{k}\right)
\end{gathered}
$$

\section{F. Modelo por envolvente compleja del canal con desvanecimiento Plano y Lento}

El modelo propuesto para analizar la modulación M-QAM bajo las condiciones de un canal Gaussiano limitado en banda y un canal con desvanecimiento plano y lento tipo Rayleigh por medio de la técnica de la envolvente compleja se ilustra en la Fig. 3.
FIG. 3. MODELO DE SIMULACIÓN M-QAM

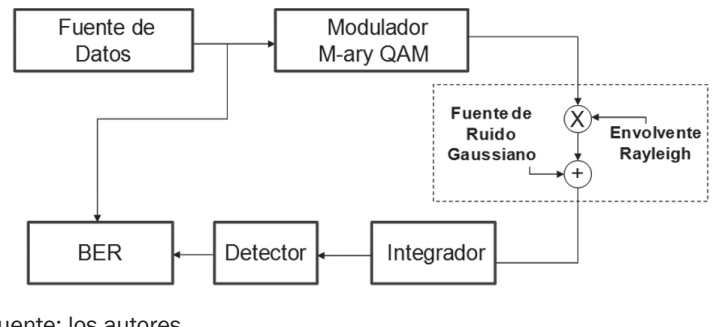

El diagrama en bloques que modela el desvanecimiento plano y lento se presenta en la Fig. 4, la cual hace uso del equivalente en frecuencia derivado de la ecuación (9). Por medio de esta implementación, la envolvente Rayleigh emula el movimiento lento y plano considerado el efecto Doppler presentado en este trabajo y que presentará el caso usual de un sistema de comunicaciones inalámbricas en donde no existen componentes dominantes de potencia entre el transmisor y el receptor [17]. La envolvente Rayleigh resultante, denotada por $\zeta(\mathrm{t})$, afecta multiplicativamente la envolvente compleja como se ilustra en la Fig. 4.

FIG. 4. MODELO DE GENERACIÓN DE LA ENVOLVENTE RAYLEIGH

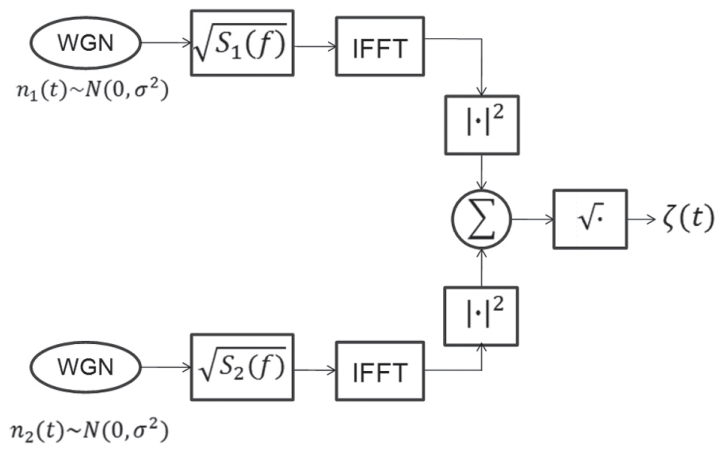

Fuente: Los autores

\section{G. Simulación por la envolvente Compleja}

Para realizar el análisis del sistema Mary QAM propuesto, se utilizó un muestreo sobre la señal en la representación de la envolvente compleja en el intervalo de tiempo $\left[k T_{s},(k+1) T_{s}\right]$. La cantidad de muestras escogidas en un periodo de un símbolo fue de ocho $(M=8)$, la cual garantiza el cumplimiento del criterio de Nyquist por símbolo.

Para la implementación del integrador en el demodulador basado en el correlator, se utilizó la definición de la integral por medio de sumas de Reiman (12), con $\Delta T=T / M$. 


$$
\int_{k T_{s}}^{(k+1) T_{s}} f(t) d t \approx \Delta T \sum_{i=0}^{M-1} f\left(k T_{s}+\Delta T \cdot i\right)
$$

Para el detector se implementó el criterio de máxima verosimilitud (ML), y como fuente, se utilizó una secuencia seudoaleatoria equiprobable con una asignación Gray de los símbolos en la constelación. Para la estimación de las curvas de desempeño de la probabilidad de error de un bit se utilizó la simulación de Montecarlo, en la cual de $N$ símbolos enviados se cuentan los $N_{e}$ símbolos recibidos que están errados. La estimación de dicha probabilidad de error se obtuvo por medio de (13).

$$
P_{B}=\frac{N_{e}}{N}
$$

Para la generación de las curvas de desempeño por medio de la simulación, se generó una secuencia equiprobable conformada por 64.000 símbolos con una velocidad de 8.000 baudios en la representación de la envolvente compleja. El estimador de la probabilidad de error bajo estas condiciones presentó un intervalo de confianza mejor al 95\%. Para determinar la relación señal a ruido en las simulaciones del canal variante en el tiempo se utilizó (14).

$$
\frac{d^{2}{ }_{\min }}{\sigma^{2}}=\frac{E\left(R^{2}\right) E_{s}}{N_{0} / 2}
$$

\section{RESULTADOS}

\section{A. Validación del modelo}

Con el objeto de validar el código desarrollado, en la Fig. 5 se detalla las curvas de error versus la relación Signal to Noise medido en decibles (SNR[dB]) para las modulaciones BPSK (2-QAM), 4-QAM, 16-QAM y 64-QAM en un canal Gaussiano limitado en banda. En esta figura se detalla la correspondencia entre la simulación y los datos teóricos de este tipo de canales.

La cantidad de símbolos utilizados en la simulación no permite encontrar valores confiables en un canal AWGN para $\mathrm{SNR}>8 \mathrm{~dB}$, debido a que se encuentra el fenómeno de eventos raros. Este fenómeno se presenta en la simulación de Monte Carlo cuando el espacio de muestra no es representativo para cuantificar los eventos de análisis. En la simulación realizada, sería posible incrementar la ventana de análisis de SNR para el caso AWGN a costa de incrementar el tiempo de computación de forma exponencial.

En la Fig. 6, se ilustra el desempeño de la modulación B-PSK (2-QAM), 4-QAM, 16-QAM y 64-QAM considerado un canal con desvanecimiento plano y lento tipo Rayleigh ideal con $E\left(R^{2}\right)=1$, habida cuenta del modelo de la Fig. 4. En esta gráfica se detalla el modelo aproximado teórico planteado en [18]-[19] versus la simulación realizada en este documento.

La comparación detallada entre los resultados obtenidos entre el canal AWGN y el canal con desvanecimiento plano y lento tipo Rayleigh ideal, permite concluir que a diferencia del canal Gaussiano limitado en banda, las curvas de error en un canal Rayleigh varían casi linealmente con respecto a la relación señal a ruido, y por eso, no es posible fácilmente mejorar la probabilidad de error del sistema con un incremento de la potencia del sistema transmisor. El cálculo teórico del desempeño de las modulaciones 16-QAM y 64-QAM planteado por [20] ante un canal con desvanecimiento plano y lento tipo Rayleigh se realizó en este trabajo por medio de integración numérica, con exigente requerimiento de recursos de computación en comparación con los requeridos para esa simulación, con un PC Pentium IV con 512 de RAM.

\section{B. Validación del Canal Rayleigh con Efecto Doppler}

Con el objeto de validar la distribución de probabilidad de la envolvente Rayleigh planteada en el modelo Jakes desarrollado, se grafica en la Fig. 7 el histograma para cada frecuencia máxima Doppler $f_{\max }$, se verificó por medio del estimador de máxima verisimilitud que la envolvente sigue una distribución de probabilidad Rayleigh. En la Fig. 8, se ilustra una realización de las variaciones de la envolvente Rayleigh en el tiempo con respecto a la máxima frecuencia Doppler para los efectos 


\section{Curvas de Desempeño para el Canal Rayleigh Dependiente de la Frecuencia Doppler}

Finalmente, en la Fig. 9 se presenta las curvas de desempeño de la modulación M-QAM incluido el desempeño bajo un canal Gaussiano limitado en banda, un canal tipo Rayleigh ideal y un canal Rayleigh con el modelo de Jackes. Para los efectos visuales se detalló las medidas de desempeño para $E\left(R^{2}\right)=0.5$ y $E\left(R^{2}\right)=1$. En dichas gráficas, se puede detallar que el caso Rayleigh ideal es la cota inferior del desempeño de la modulación bajo el supuesto de un canal variante con el tiempo lento y plano que modele el fenómeno Doppler descrito en este documento.

\section{CONCLUSIONES}

En el presente artículo se presentó la metodología de análisis y las curvas de desempeño de la modulación M- QAM en un canal AWGN limitado en banda y en un canal con desvanecimiento plano y lento tipo Rayleigh, tanto ideal como con un modelo de desvanecimiento en frecuencia, por medio de la teoría de la envolvente compleja. Las curvas de la probabilidad de error encontradas para el caso del canal con desvanecimiento plano y lento tipo Rayleigh muestran un aumento considerable en la probabilidad de error en comparación de un canal AWGN limitado en banda.

FIG. 5. PROBABILIDAD DE ERROR PARA M-QAM EN UN CANAL AWGN.

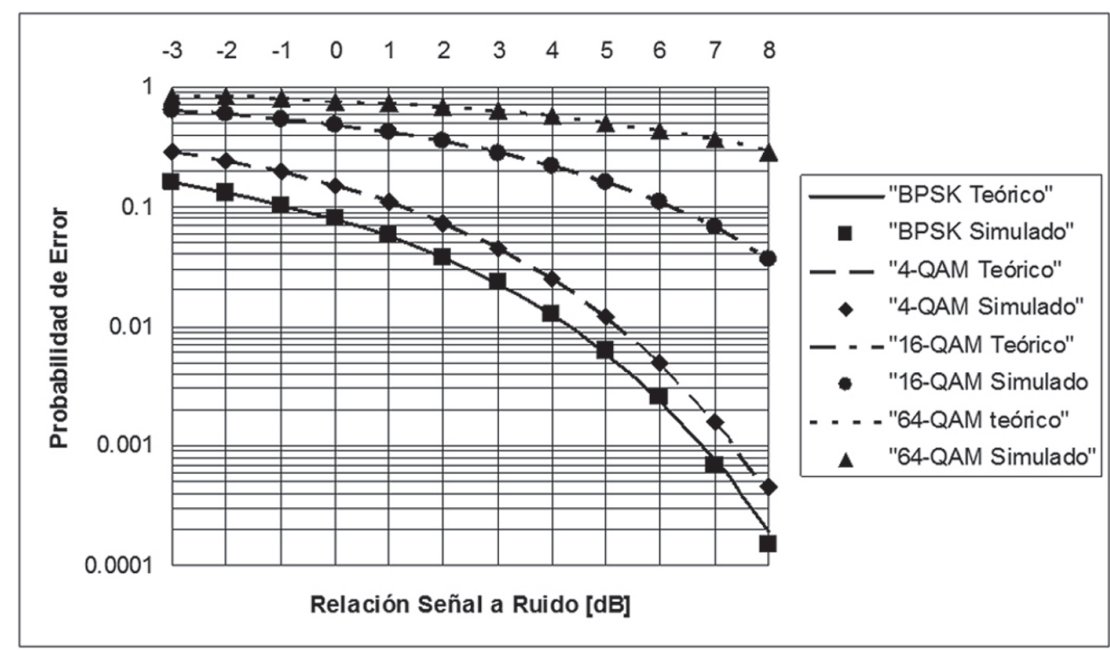

Fuente: los autores

FIG. 6. PROBABILIDAD DE ERROR PARA M-QAM EN UN CANAL CON DESVANECIMIENTO PLANO Y LENTO TIPO RAYLEIGH.

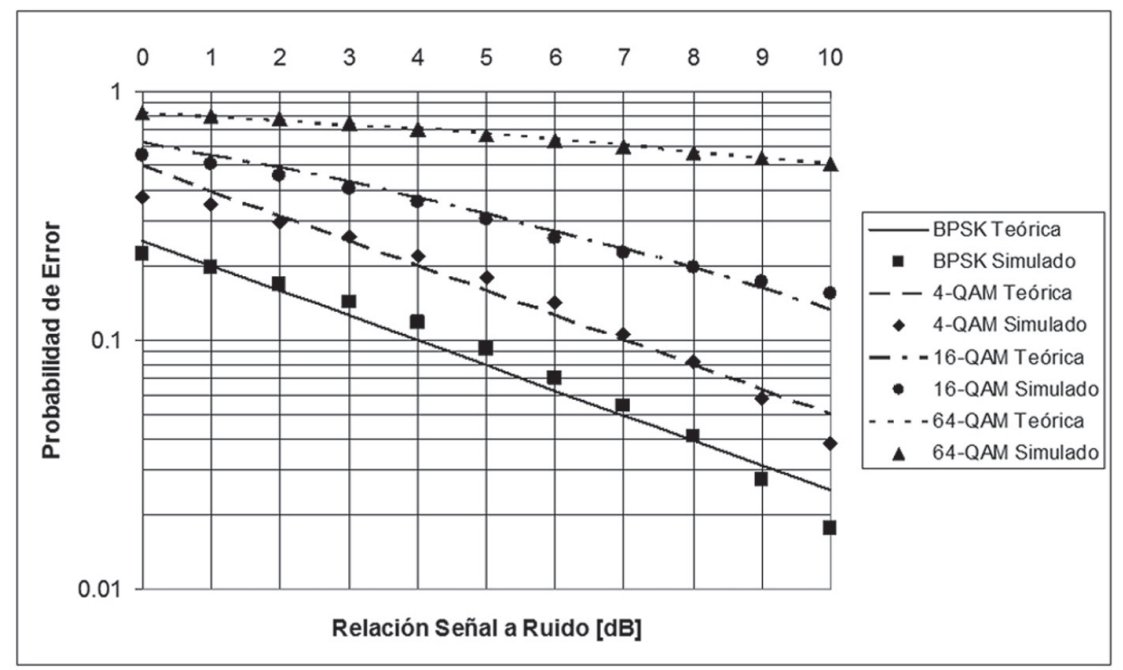


FIG. 7. FUNCIÓN DE DENSIDAD DE PROBABILIDAD EXPERIMENTAL PARA LA ENVOLVENTE RAYLEIGH CON RESPECTO A LAS VARIACIONES DE $f_{\text {max }}$.
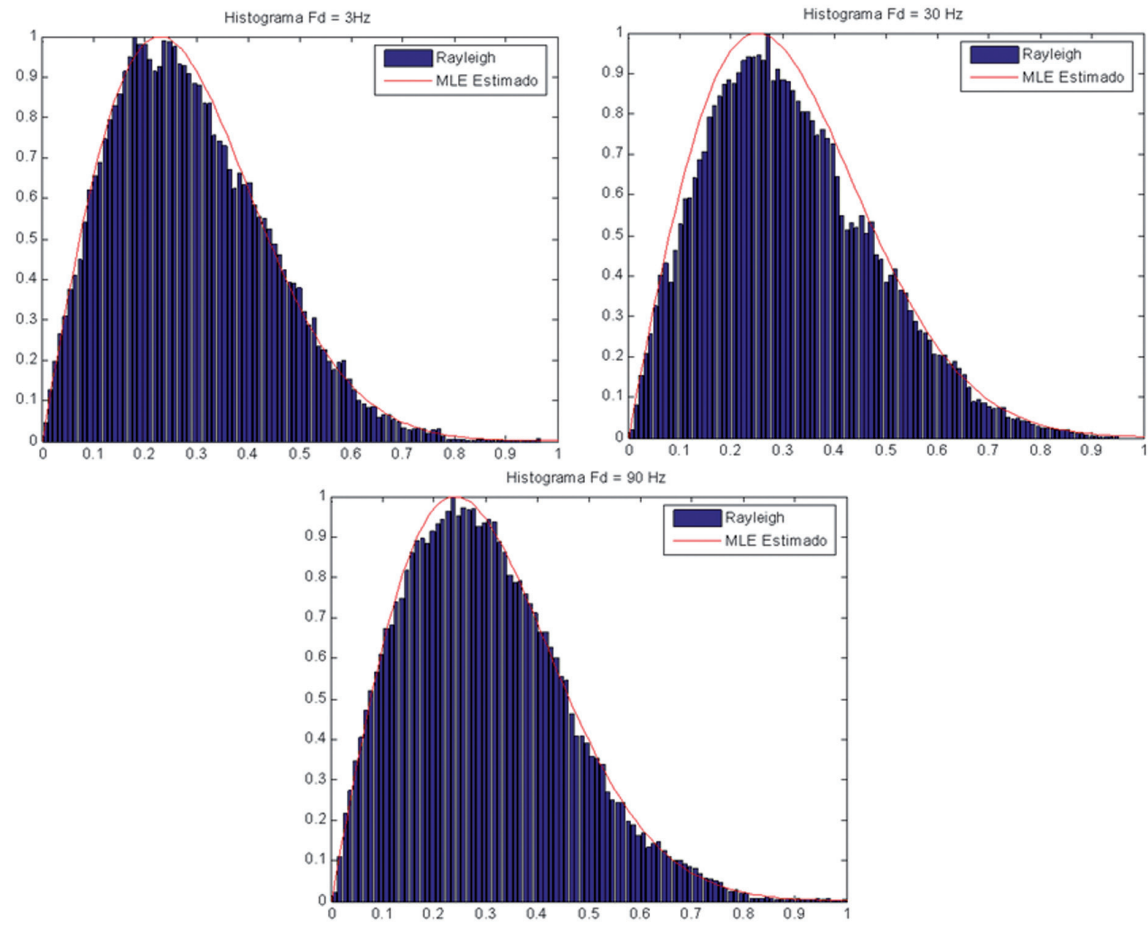

Fuente: los autores

FIG. 8. ENVOLVENTE RAYLEIGH CON RESPECTO A LAS VARIACIONES DE $f_{\max }$.
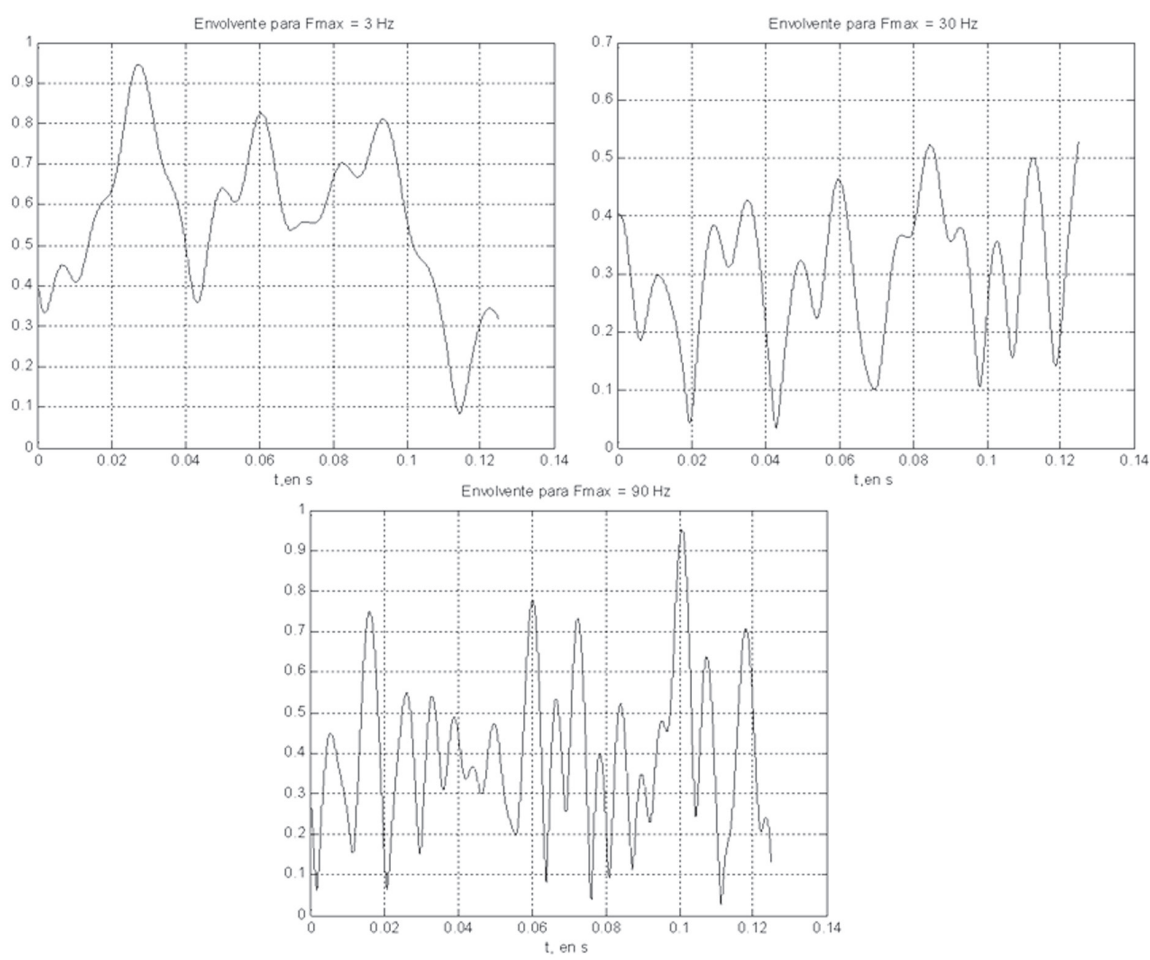

Fuente: Los autores 
FIG. 9. DESEMPEÑO DE LA MODULACIÓN M-ARY INCLUYENDO EL MODELO DE JAKES. FUENTE: AUTOR DEL PROYECTO.
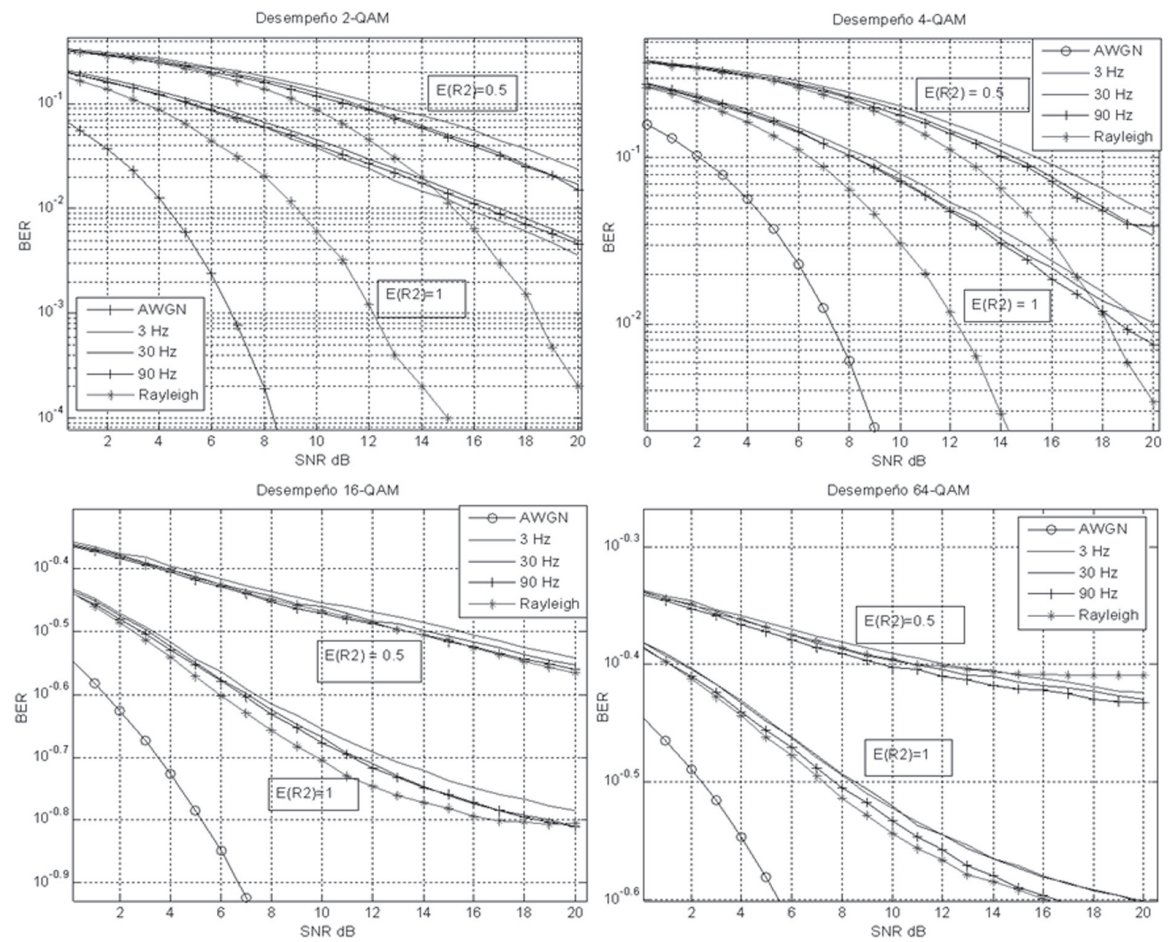

Fuente: Los autores

Ya que el canal con desvanecimiento plano y lento tipo Rayleigh es un canal variante en tiempo usual en aplicaciones inalámbricas, en la cual no existe línea de vista entre el transmisor y el receptor, la metodología planteada es valiosa para encontrar las medidas de desempeño de un sistema que puede tener una difícil descripción analítica. Este es el caso de las nuevas tecnologías inalámbricas, como los estándares LTE (Long Term Evolution) y televisión digital DVB-T, cuyos esquemas de modulación avanzados se basan en la modulación M-QAM.En un canal de características variantes en tiempo, como el analizado, la medida de desempeño de la probabilidad de error no puede ser mejorada según la estrategia tradicional de incremento de la energía por símbolo en el transmisor, ya que las curvas de desempeño encontradas muestran una mejora casi lineal, comparada con la mejora de dicha medida de desempeño de tipo exponencial en un canal Gaussiano limitado en banda. Este resultado implica, que se deben buscar estrategias alternativas a incrementar la potencia, si se desea tener una mejora representativa en la calidad del enlace de comunicaciones.

El modelo de Jakes, aunque oportuno y simplificado para modelar un canal con desvanecimiento plano y lento no tiene vinculado explícitamente la relación entre la frecuencia Doppler máxima de un modelo de propagación específico. En la utilización de este canal, la literatura usualmente especifica los parámetros del canal como una característica del modelo a pequeña escala, y por tal razón, un parámetro de características locales. Este es el mayor inconveniente en la utilización racional de este modelo y, por eso, requiere un mayor estudio, que permita darle una mayor relación con un entorno real de radio propagación.

\section{AGRADECIMIENTOS}

Los autores desean dar sus agradecimientos a la Pontificia Universidad Javeriana y al Departamento de Electrónica de dicha universidad, por facilitar los recursos necesarios de Software, Hardware y personal para realizar a cabo el presente trabajo. 


\section{REFERENCIAS}

[1] E. Biglieri, J. Proakis, and S. Shamani, "Fading Channels: Information-Theoretic and Communications Aspects," IEEE Transactions on Information Theory, vol.44, no.6, pp.2619-2692, Oct 1998

[2] K. Feher, Wireless Digital Communications: Modulation and Spread Spectrum Applications, May 1995

[3] W. Webb and R. Steele, "Variable rate QAM for mobile radio", IEEE Transactions on Communications, vol.43, no.7, pp.2223-2230, Jul 1995

[4] S. Benedetto and E. Biglieri, Principles of Digital Transmission: With Wireless Applications, Kluwer Academic Pub, 1999

[5] S. Haykin. Communications Systems, Fourth Edition, John Willey \& Sons, Inc, 2002

[6] J. Proakis, Digital Communications, Mc Graw Hill 2004

[7] K.S. Shanmugan, "Simulation and Implementation Tools for Signal Processing and Communication Systems," IEEE Communications Magazine, vol.32, no.7, pp.36-40, Jul 1994

[8] W.H. Tranter and K.L. Kosbar, "Simulation of Communication Systems," Communications Magazine, IEEE , vol.32, no.7, pp.26-35, Jul 1994

[9] B. Woerner, J.H. Reed and T. Rappaport, "Simulation Issues for Future Wireless Modems," IEEE Communications Magazine, vol.32, no.7, pp.4253, Jul 1994

[10] A. Goldsmith, Wireless Communications, Cambridge University Press 2005

[11] T.S. Rappaport. Wireless Communications, Prentice Hall Communications Engineering and Emerging Technologies Series, New Jersey, 1996

[12] E. Biblieri, "Digital transmission in the 21st Century: Conflating Modulation and Coding", Communications Magazine, IEEE , vol.40, no.5, pp.128-137, May 2002

[13] D.J. Gibson, The Communications Handbook, Editor in Chief. Second Edition CRC Press, 2002

[14] B. Sklar, "Rayleigh Fading Channels in Mobile Digital Communication Systems I. Characteriza- tion," IEEE Communications Magazine, vol.35, no.7, pp.90-100, Jul 1997

[15] M. Pätzold, R. Garcia and F. Laue, "Design of High-Speed Simulation Models for Mobile Fading Channels by Using Table Look-Up Techniques," IEEE Transactions on Vehicular Technology, vol.49, no.4, pp.1178-1190, Jul 2000

[16] R. H. Clarke, "A Statistical Theory of Mobile Radio Reception," Bell Systems Technical Journal, Vol. 47 pp. 957 - 1000, 1968

[17] W. Tranter, K.S. Shanmugan, T. Rappaport and K. Kosbar, Principles of Communication Systems Simulation with Wireless Applications, Prentice Hall. 2004

[18] C.A. Gutierrez, M. Cabrera and J. Sanchez, "Generation of correlated rayleigh-fading envelopes for simulating the variant behavior of indoor radio propagation channels," Vehicular Technology Conference, vol.6, no., pp. 4245- 4249, Sept, 2004

[19] L. Hanzo, W. Webb, T. Keller. Single and MultiCarrier Quadrature Amplitude Modulation: Principles and Applications for Personal Communcations, WLANs and Broadcasting. Willey Chichester, 2000

[20] X. Tang, M. Alouini, and A. Goldsmith, "Effect of Channel Estimation Error on M-QAM BER Performance in Rayleigh Fading", IEEE Transactions on Communications, vol.47, no.12, pp.1856-1864, Dec 1999. 